\title{
Direct controller order reduction by identification in closed loop
}

\author{
Ioan Doré Landau ${ }^{\mathrm{a}}$, Alireza Karimi ${ }^{\mathrm{b}}$, Aurelian Constantinescu ${ }^{\mathrm{c}}$ \\ ${ }^{\text {a }}$ Laboratoire d'Automatique de Grenoble,ENSIEG, BP 46 \\ 38402 Saint Martin d'Hères, France \\ ${ }^{\mathrm{b}}$ Sharif University of Technology, Electrical Engineering Dept., \\ Tehran 11365-9363, IRAN \\ ${ }^{\mathrm{c}}$ Laboratoire d'Automatique de Grenoble,ENSIEG, BP 46 \\ 38402 Saint Martin d'Hères, France
}

\begin{abstract}
The paper ${ }^{1}$ addresses the problem of directly estimating the parameters of a reduced order digital controller using a closed loop type identification algorithm. The algorithm minimizes the closed loop plant input error between the nominal closed loop system and the closed loop system using the reduced order controller. It is assumed that a plant model (if necessary validated in closed loop with the nominal controller) is available. One of the original features of this approach is that it can use either simulated or real data. The frequency bias distribution of the parameter estimates shows that the reduced order controller maintains the critical performance of the nominal closed loop system. A theoretical analysis is provided. Validation tests are proposed. Experimental results, obtained on an active suspension, illustrate the performance of the proposed algorithms.
\end{abstract}

Key words: Controller reduction, closed loop identification, active suspension.

1 A preliminary version of this paper has been presented to the IFAC-SYSID Symposium, June 2000, S. Barbara, USA

* Corresponding author I. D. Landau. Fax 33(0)4.76.82.63.88.

E-mail landau@lag.ensieg.inpg.fr Tel. 33(0)4.76.82.63.91.

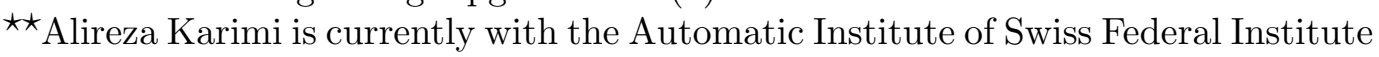
of Technology at Lausanne (EPFL).

Email addresses: alireza.karimi@epfl.ch (Alireza Karimi), aurelian@lag.ensieg.inpg.fr (Aurelian Constantinescu). 


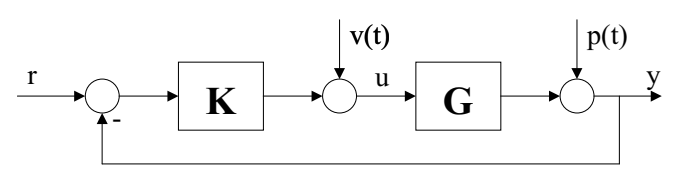

Fig. 1. The closed loop system

\section{Notations}

Consider the system shown in Fig. 1 where the plant model transfer function is given by:

$$
G\left(z^{-1}\right)=\frac{z^{-d} B\left(z^{-1}\right)}{A\left(z^{-1}\right)}
$$

and the nominal controller by:

$$
K\left(z^{-1}\right)=\frac{R\left(z^{-1}\right)}{S\left(z^{-1}\right)}
$$

The following sensitivity functions are defined:

- output sensitivity function

$$
S_{y p}\left(z^{-1}\right)=\frac{1}{1+K G}=\frac{A\left(z^{-1}\right) S\left(z^{-1}\right)}{P\left(z^{-1}\right)}
$$

- input sensitivity function

$$
S_{u p}\left(z^{-1}\right)=-\frac{K}{1+K G}=-\frac{A\left(z^{-1}\right) R\left(z^{-1}\right)}{P\left(z^{-1}\right)} ;
$$

- output sensitivity function with respect to an input disturbance

$$
S_{y v}\left(z^{-1}\right)=\frac{G}{1+K G}=\frac{z^{-d} B\left(z^{-1}\right) S\left(z^{-1}\right)}{P\left(z^{-1}\right)}
$$

- complementary sensitivity function

$$
S_{y r}\left(z^{-1}\right)=\frac{K G}{1+K G}=\frac{z^{-d} B\left(z^{-1}\right) R\left(z^{-1}\right)}{P\left(z^{-1}\right)},
$$

where

$$
P\left(z^{-1}\right)=A\left(z^{-1}\right) S\left(z^{-1}\right)+z^{-d} B\left(z^{-1}\right) R\left(z^{-1}\right)
$$


The system of Fig.1 will be denoted the "true closed loop system". Throughout the paper we will consider feedback systems which will use either an estimation of $G$ (denoted $\hat{G}$ ) or a reduced order estimation of $K$ (denoted $\hat{K})$. The corresponding sensitivity functions will be denoted as follows:

- $S_{x y}$ - Sensitivity function of the true closed loop system $(K, G)$.

- $\hat{S}_{x y}$ - Sensitivity function of the nominal simulated closed loop system (nominal controller $K+$ estimated plant model $\hat{G}$ ).

- $\hat{\hat{S}}_{x y}$ - Sensitivity function of the simulated closed loop system using a reduced order controller (reduced controller $\hat{K}+$ estimated plant model $\hat{G}$ ).

Similar notations are used for $P\left(z^{-1}\right): \hat{P}\left(z^{-1}\right)$ when using $K$ and $\hat{G}, \hat{\hat{P}}\left(z^{-1}\right)$ when using $\hat{K}$ and $\hat{G}$.

\section{Introduction}

Controller design frequently results in high order controllers. On one hand this may be the consequence of the complexity of the model used for design, on the other hand robust controller design often results in complex high order controllers even if the design model is of reasonable size. See for example Landau et al. (1995).

Controller reduction is a very important issue in many control applications either because the size of the controller is limited by hardware and computation time or because simpler controllers are easier to implement and to understand. There are a number of approaches and methods for obtaining reduced order controllers. See for example Zhou and Doyle (1998); Anderson (1993); Anderson and Liu (1989).

What is most important is to remember that controller reduction should aim to preserve the required closed loop properties as far as possible. Direct simplification of the controller using standard techniques (pole-zero cancellation within a certain radius, balanced reduction) without taking into account the closed loop behaviour generally yields unsatisfactory results.

There are basically two approaches for controller reduction:

(1) Indirect approach:

- Obtain a reduced order model which will capture the essential characteristics of the nominal model in the critical frequency regions for design.

- Design a new controller using the new low-order model.

(2) Direct approach: 


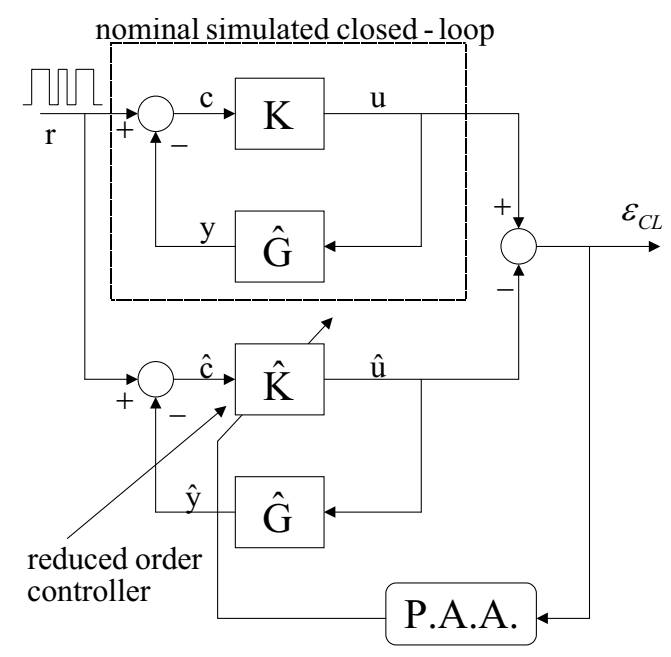

Fig. 2. Closed loop identification of reduced order controllers using simulated data (input matching)

- Obtain an approximate reduced order controller which will preserve the nominal closed loop properties.

The indirect approach is subject to a number of criticisms. First of all, use of a reduced order model does not necessarily guarantee that the resulting controller will be of a sufficiently low order (design specifications usually become more complex when using model approximation). Secondly, the errors caused by model approximation will spread to the subsequent design steps, see Anderson and Liu (1989); Cordons et al. (1999).

The direct approach to controller reduction seems more appropriate because the approximation is carried out in the final step of controller design and the results can be easily understood. It should be noted that the controller resulting from an indirect reduction procedure can be further reduced, if necessary, by application in the last step of a direct reduction approach.

Identification in closed loop offers an efficient methodology both for model order reduction and direct controller order reduction (see Bendotti et al. (1998)).

In this paper we will focus on the use of closed loop identification techniques for direct controller order reduction. One of the basic block diagrams for reduced order controller identification is shown in Fig. 2 (input matching scheme ). The upper part represents the simulated nominal closed loop system. It is made up of the nominal designed controller (denoted by $K$ ) and the best identified plant model (i.e which results in the closest behaviour of the true closed loop system and of the nominal simulated one and denoted by $\hat{G}$ ).

The lower part is made up of the estimated reduced order controller (denoted 
by $\hat{K}$ ) in feedback connection with the plant model used for simulation of the nominal system. The parametric adaptation algorithm will try to find the best reduced order controller of a given order which will minimize the closed loop input error expressed as the difference between the input to the plant model generated in the nominal simulated closed loop and the input to the plant model generated by the closed loop using the reduced order controller (i.e which will minimize the discrepancy between the two closed loops).

However, another objective for controller order reduction can be to minimize the closed loop error between the plant output generated in the nominal simulated closed loop and the plant output generated by the closed loop using the reduced order controller. As will be shown, this is possible using either the scheme of Fig. 2 but filtering the external excitation $r$ through $\hat{G}$ or by adding the external excitation to the input of the plant instead of to the input of the controller (in both cases the closed loop error, in the absence of disturbances, will reflect the difference between the two plant model outputs).

Identification of a reduced order controller is also possible using real data as shown in Fig. 3 (the upper part represents the true closed loop system ). Note that the adjustable closed loop predictor (lower part) may use a plant model identified in closed loop with the nominal controller or more precisely the plant model available which yields the best results when a "closed loop" model validation test is used (see Landau and Karimi (1997a); Landau et al. (1997)). In short, this plant model will minimize the discrepancy between the nominal true closed loop system and the simulated closed loop system using the nominal controller.

The method for direct controller reduction using real data may be related to iterative feedback tuning (IFT) in Hjalmarsson et al. $(1998,1994)$. The main differences are:(1)the IFT requires several experiments;(2) the IFT does not use an estimated model of the plant to tune a reduced order controller.

The paper is organized as follows. In Section 2 some basic relationships considered in controller reduction will be reviewed. In Section 3 the recursive algorithms for direct identification of the reduced order controllers will be presented and analyzed. Validation of the estimated reduced order controller will be discussed in Section 4. The practical aspects of the methodology will be summarized in Section 5. Experimental results concerning the identification of reduced order controllers for active suspension will be given in Section 6 . Finally we will have some concluding remarks. 


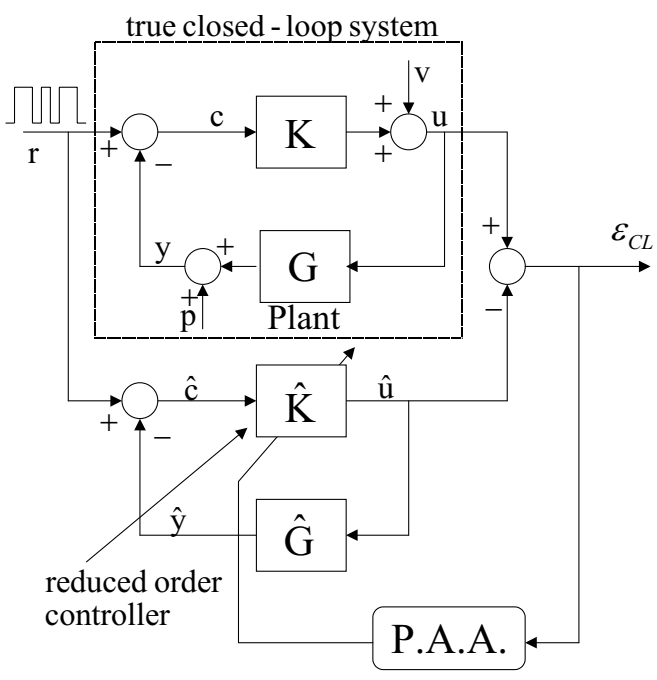

Fig. 3. Closed loop identification of the reduced order controllers using real data (input matching)

\section{Direct Controller Reduction - Some basic facts}

Several criteria for controller reduction have been proposed by Anderson (1993); Zhou and Doyle (1998). Their objective is the minimization of the discrepancy between the nominal closed loop and the loop using the reduced order controller. Let us consider the block diagram of Fig. 2. Consider the "input matching" objective which aims to preserve performance with respect to the effect of the output disturbance upon the plant input by minimization of the closed loop error between the two plant inputs $u$ and $\hat{u}$. This is equivalent to minimizing the following norm:

$$
\left\|\hat{S}_{u p}-\hat{\hat{S}}_{u p}\right\|=\left\|\frac{K}{1+K \hat{G}}-\frac{\hat{K}}{1+\hat{K} \hat{G}}\right\|
$$

where $\hat{S}_{u p}$ is the input sensitivity function of the nominal simulated closed loop and $\hat{\hat{S}}_{u p}$ is the input sensitivity function when using the reduced order controller. Therefore the optimal reduced order controller will be given by:

$$
\hat{K}^{*}=\arg \min _{\hat{K}}\left\|\hat{S}_{u p}-\hat{\hat{S}}_{u p}\right\|=\arg \min _{\hat{K}}\left\|\hat{S}_{y p}(K-\hat{K}) \hat{\hat{S}}_{y p}\right\|
$$

If we now consider preservation of performance in tracking, the reduced order controller should minimize the following norm:

$$
\left\|\hat{S}_{y r}-\hat{\hat{S}}_{y r}\right\|=\left\|\frac{K \hat{G}}{1+K \hat{G}}-\frac{\hat{K} \hat{G}}{1+\hat{K} \hat{G}}\right\|
$$


In the same way as when preserving performance for output disturbance rejection, the reduced order controller should minimize:

$$
\left\|\hat{S}_{y p}-\hat{\hat{S}}_{y p}\right\|=\left\|\frac{1}{1+K \hat{G}}-\frac{1}{1+\hat{K} \hat{G}}\right\|
$$

Fortunately, these two norms are equal and the reduced order controller can be obtained by the following expression:

$$
\hat{K}^{*}=\arg \min _{\hat{K}}\left\|\hat{S}_{y p}-\hat{\hat{S}}_{y p}\right\|=\arg \min _{\hat{K}}\left\|\hat{S}_{y p}(K-\hat{K}) \hat{\hat{S}}_{y v}\right\|
$$

Eqs. (5) and (8) show that a weighted norm of $K-\hat{K}$ should be minimized. Several methods for solving this problem have been proposed in the literature. The model reduction using weighted balanced realization in Enns (1984) solves an approximation of the problem, i.e. it neither minimizes the infinity norm of the weighted error nor gives the error bounds. Another approach proposed by Anderson (1986) minimizes the Hankel norm of the weighted error as well as giving the error bounds.

In this paper we present an approach for minimization of the 2-norms considered in (5) and (8) by direct identification of a reduced controller in closed loop. The identification algorithm will minimize a 2-norm of a closed loop prediction error. We will show that the modeling error between the nominal and reduced order controller is exactly weighted with the filters of Eq. (5) or (8). Unstable parts and fixed parts of the controller, which should be preserved in the reduced order controller, can be easily treated. It is assumed that a stabilizing controller of a given complexity exists and that the algorithm will search the parameters of this controller. Once such a reduced order controller is obtained, it should be validated and validation tests are proposed. The Vinnicombe distance between the two closed loop transfer functions (the nominal closed loop and the closed loop using the reduced order controller) provides useful information on the quality of the approximation. What is certainly very interesting and introduces new features for the proposed procedure is that our presentation shows that the reduced order controller can be identified using real data.

\section{Algorithms for direct closed loop identification of reduced order controllers}

The parametric adaptation algorithms which will be used to identify the parameters of a reduced order controller are very similar to the "closed loop out- 
put error" (CLOE) algorithms used for plant model identification in closed loop. To a large extent, the problem of identification of reduced order controllers can be considered to be the dual of the reduced order plant model identification in closed loop (Landau and Karimi (1997a,b); Landau et al. (1997); Landau and Karimi (2000)). We will often refer to Landau and Karimi (1997a,b); Landau et al. (1997) for details of the algorithms and proofs.

\subsection{Algorithms}

Consider the upper part of Fig. 2. The nominal simulated closed loop is formed by the estimated plant model and the nominal controller. The estimated plant model is defined by the transfer operator $\left(q^{-1}-\right.$ unit delay operator):

$$
\hat{G}\left(q^{-1}\right)=\frac{q^{-d} \hat{B}\left(q^{-1}\right)}{\hat{A}\left(q^{-1}\right)}
$$

where:

$$
\begin{aligned}
& \hat{B}\left(q^{-1}\right)=\hat{b}_{1} q^{-1}+\cdots+\hat{b}_{n_{B}} q^{-n_{B}}=q^{-1} \hat{B}^{*}\left(q^{-1}\right) \\
& \hat{A}\left(q^{-1}\right)=1+\hat{a}_{1} q^{-1}+\cdots+\hat{a}_{n_{A}} q^{-n_{A}}=1+q^{-1} \hat{A}^{*}\left(q^{-1}\right) .
\end{aligned}
$$

The plant model is operated in closed loop with a digital controller:

$$
K=\frac{R\left(q^{-1}\right)}{S\left(q^{-1}\right)}
$$

where:

$$
\begin{aligned}
R\left(q^{-1}\right) & =r_{0}+r_{1} q^{-1}+\ldots+r_{n_{R}} q^{-n_{R}} \\
S\left(q^{-1}\right) & =1+s_{1} q^{-1}+\ldots+s_{n_{s}} q^{-n_{S}}=1+q^{-1} S^{*}\left(q^{-1}\right)
\end{aligned}
$$

$u(t)$ is the plant input, $y(t)$ is the plant output and $r(t)$ is the external excitation signal (filtered if necessary).

The output of the nominal controller is given by:

$$
u(t+1)=-S^{*}\left(q^{-1}\right) u(t)+R\left(q^{-1}\right) c(t+1)=\theta^{T} \psi(t)
$$

where 


$$
\begin{aligned}
c(t+1) & =r(t+1)-y(t+1) \\
y(t+1) & =-\hat{A}^{*} y(t)+\hat{B}^{*} u(t-d) \\
\psi^{T}(t) & =\left[-u(t), \ldots,-u\left(t-n_{S}+1\right), c(t+1), \ldots, c\left(t-n_{R}+1\right)\right] \\
\theta^{T} & =\left[s_{1}, \ldots, s_{n_{S}}, r_{0}, \ldots, r_{n_{R}}\right]
\end{aligned}
$$

To implement and analyze the algorithm, we need respectively the a priori (based on $\hat{\theta}(t)$ ) and the a posteriori (based on $\hat{\theta}(t+1)$ ) predicted outputs of the estimated reduced order controller (of orders $n_{\hat{S}}$ and $n_{\hat{R}}$ ) which are given by (see the lower part of Fig. 2):

a priori:

$$
\begin{aligned}
\hat{u}^{0}(t+1) & =\hat{u}(t+1 \mid \hat{\theta}(t))=-\hat{S}^{*}\left(t, q^{-1}\right) \hat{u}(t)+\hat{R}\left(t, q^{-1}\right) \hat{c}(t+1) \\
& =\hat{\theta}^{T}(t) \phi(t)
\end{aligned}
$$

a posteriori:

$$
\hat{u}(t+1)=\hat{\theta}^{T}(t+1) \phi(t)
$$

where:

$$
\begin{aligned}
& \hat{\theta}^{T}(t)=\left[\hat{s}_{1}(t), \ldots, \hat{s}_{n_{\hat{S}}}(t), \hat{r}_{0}(t), \ldots, \hat{r}_{n_{\hat{R}}}(t)\right] \\
& \phi^{T}(t)=\left[-\hat{u}(t), \ldots,-\hat{u}\left(t-n_{\hat{S}}+1\right), \hat{c}(t+1), \ldots, \hat{c}\left(t-n_{\hat{R}}+1\right)\right] \\
& \hat{c}(t+1)=r(t+1)-\hat{y}(t+1)=r(t+1)+\hat{A}^{*} \hat{y}(t)-\hat{B}^{*} \hat{u}(t-d)
\end{aligned}
$$

\subsubsection{Closed loop input matching algorithm (CLIM)}

The closed loop input error is given by:

a priori:

$$
\varepsilon_{C L}^{0}(t+1)=u(t+1)-\hat{u}^{0}(t+1)
$$

a posteriori:

$$
\varepsilon_{C L}(t+1)=u(t+1)-\hat{u}(t+1)
$$

and the parameter adaptation algorithm will be given by: 


$$
\begin{aligned}
& \hat{\theta}(t+1)=\hat{\theta}(t)+F(t) \Phi(t) \varepsilon_{C L}(t+1) \\
& F^{-1}(t+1)=\lambda_{1}(t) F^{-1}(t)+\lambda_{2}(t) \Phi(t) \Phi^{T}(t) \\
& 0<\lambda_{1}(t) \leq 1 ; 0 \leq \lambda_{2}(t)<2 ; \quad F(0)>0 \\
& \varepsilon_{C L}(t+1)=\frac{\varepsilon_{C L}^{0}(t+1)}{1+\Phi^{T}(t) F(t) \Phi(t)}=\frac{u(t+1)-\hat{u}^{0}(t+1)}{1+\Phi^{T}(t) F(t) \Phi(t)}
\end{aligned}
$$

As we can see from (29), the a posteriori closed loop input error $\varepsilon_{C L}(t+1)$ can be expressed in terms of the a priori (measurable) closed loop input error $\varepsilon_{C L}^{0}(t+1)$. Therefore the right hand side of (27) will depend only on measurable quantities at $t+1$. For more details see Landau et al. (1997).

Specific algorithms will be obtained by an appropriate choice of observation vector $\Phi(t)$ as follows:

- CLIM: $\Phi(t)=\phi(t)$

- F-CLIM: $\Phi(t)=\frac{\hat{A}\left(q^{-1}\right)}{\hat{P}\left(q^{-1}\right)} \phi(t)$

where:

$$
\hat{P}\left(q^{-1}\right)=\hat{A}\left(q^{-1}\right) S\left(q^{-1}\right)+q^{-d} \hat{B}\left(q^{-1}\right) R\left(q^{-1}\right) .
$$

\subsubsection{Closed loop output matching algorithm (CLOM)}

The objective is to create a closed loop error which reflects the difference between the output $y(t)$ of the nominal closed loop simulated system and the output $\hat{y}(t)$ of the simulated closed loop system using the reduced order controller. Two solutions are proposed:

(1) Filtering the external excitation (CLOM1)

Consider the upper part of the Fig. 2. The output of the plant model in the absence of disturbances is given by:

$$
y(t)=\frac{K \hat{G}}{1+K \hat{G}} r(t)
$$

Now, if instead of directly using $r(t)$ we apply to the system $r(t)$ filtered through $\hat{G}$ we obtain (see Fig. 4a):

$$
\bar{u}(t)=\frac{K \hat{G}}{1+K \hat{G}} r(t)
$$

and therefore, in the absence of disturbances, the two transfer operators are the same. The situation is similar for the feedback system using the lower order controller $\hat{K}$. Therefore, if we use the CLIM algorithms but filter first $r(t)$ by $\hat{G}$, the algorithm will minimize the closed loop output error instead of the closed loop input error. 


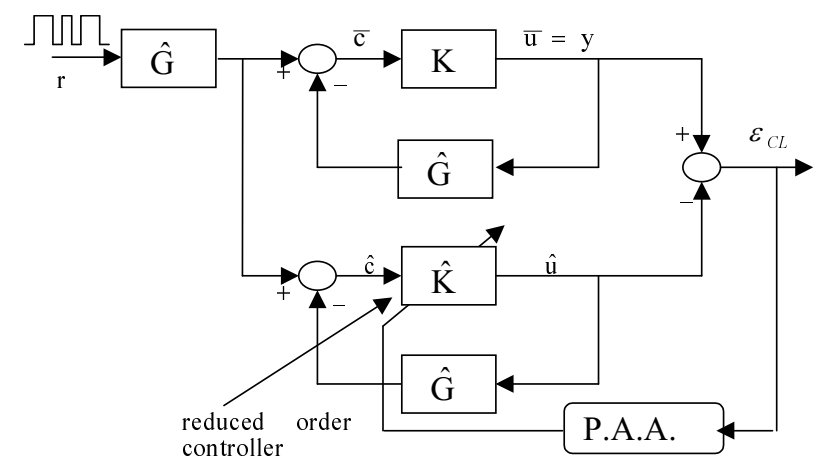

a)

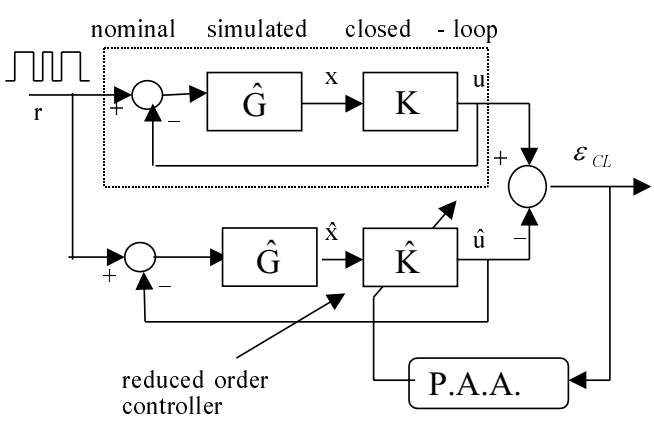

b)

Fig. 4. Closed loop output matching (CLOM): a) CLIM algorithm with filtered external excitation (CLOM1); b) CLIM algorithm with external excitation applied to the plant input (CLOM2)

(2) Adding the external excitation to the plant input (CLOM2)

This is illustrated in Fig. 4b. By changing the point of application of the external excitation, the relation between $r(t)$ and $u(t)$ is given by (32), i.e. in the absence of disturbances, the new $u(t)$ will be equal to $y(t)$. For effective implementation of the algorithm, the only changes occur in Eqs. (15) and (18) where $c(t)$ is replaced by:

$$
x(t)=\hat{G}(r(t)-\bar{u}(t))
$$

Note also that for real time experiments (as well as in simulation), the order of the blocks in the upper part of the scheme given in Fig. 4b can be interchanged.

\subsubsection{Imposing constraints on the reduced order controllers}

Without difficulty, fixed parts (filters) can be forced in the reduced order controllers (like integrators, opening of the loop at $0.5 f_{s}$, fixed filters etc.). 
For this, the nominal controller is factorized as $K=K_{F} K^{\prime}$ and the reduced order controller is factorized as $\hat{K}=K_{F} \hat{K}^{\prime}$ where $K_{F}$ corresponds to the fixed part of the controller that we would like to be contained in the reduced order controller. Then a new input to the controller $\hat{K}^{\prime}$ will be defined as $\hat{c}^{\prime}=K_{F} \hat{c}$ and $c$ is replaced by $\hat{c}^{\prime}$ in the observation vector $\Phi$.

\subsection{Stability analysis}

Using the results of the analysis given in Landau and Karimi (1997a,b); Landau et al. (1997) and by duality arguments (interchanging B and A with R and $\mathrm{S}$ respectively), it can be shown that if the estimated controller has the same structure as the nominal controller, then the closed loop error goes to zero and the signals remain bounded, provided that a sufficient strict passivity condition of the form

$$
H^{\prime}\left(z^{-1}\right)=H\left(z^{-1}\right)-\frac{\lambda}{2} ; \max _{t} \lambda_{2}(t) \leq \lambda<2
$$

is a strictly positive real transfer function is satisfied, where:

$$
H=\left\{\begin{array}{c}
\hat{A} / \hat{P} \text { for } C L I M \\
1 \text { for } F-C L I M
\end{array}\right.
$$

Strictly positive real condition also requires that for CLIM (as well as for FCLIM) $\hat{A}$ be an asymptotically stable polynomial. Note that for F-CLIM the passivity condition is always satisfied since the filters which have to be used in the algorithm contain known quantities. The same passivity conditions are valid for the CLOM algorithm.

The passivity conditions (34) and (35) also guarantee the asymptotic unbiasedness of the estimates in the presence of measurement noise (noise and external excitation are assumed to be independent).

In the case of estimation of a reduced order controller, the following assumptions are made:

- There is a reduced order controller characterized by unknown polynomials $\hat{S}$ (of order $n_{\hat{S}}$ ) and $\hat{R}$ (of order $n_{\hat{R}}$ ) which stabilizes the closed loop system.

- $r(t)$ is norm bounded.

- The output of the nominal controller (15) can be expressed as:

$$
u(t+1)=-\hat{S}^{*}\left(q^{-1}\right) u(t)+\hat{R}\left(q^{-1}\right) c(t+1)+\eta(t+1)
$$


where $\eta(t)$ is a norm bounded signal.

Equation (36) can be interpreted as a decomposition of the nominal controller into two parallel blocks: one is the reduced order controller and the other is the neglected part generating $\eta(t)$. The boundedness of $\eta(t)$ requires the neglected part to be stable. The practical consequence of this assumption is that any unstable parts of the nominal controller should remain in the reduced order controller. This can be imposed, for example, as a fixed part in the reduced order controller.

Based on the results of Landau and Karimi (1997b) (pg. 1505-1506) and assuming that $r(t)$ is norm bounded, we can show that all the signals are norm bounded under the passivity conditions (34) and (35). Therefore, when the estimated controller does not have the same structure as the nominal controller, the above conditions ensure the boundedness of the closed loop input error and closed loop output error respectively. For more details see Landau and Karimi (1997a,b).

Under the above assumptions a stable closed loop system will be obtained if the data length is long enough to allow convergence to the stabilizing controller (if there is not enough data, recycling of the data is possible).

\subsection{Bias analysis}

In the case of controller order reduction, by definition the estimated reduced order controller does not have the same structure as the nominal controller. Therefore an asymptotic bias will occur which can be characterized in the frequency domain.

We will now examine the bias for the various algorithms when using simulated and real time data.

\subsubsection{Use of simulated data}

(1) Closed loop input matching algorithm

The output of the nominal and reduced order controller can be expressed as:

$$
\begin{aligned}
& u(t)=\frac{K}{1+K \hat{G}} r(t) \\
& \hat{u}(t)=\frac{\hat{K}}{1+\hat{K} \hat{G}} r(t)
\end{aligned}
$$


The closed loop input error will be given by:

$$
\begin{aligned}
\varepsilon_{C L}(t) & =u(t)-\hat{u}(t)=\left[\frac{K}{1+K \hat{G}}-\frac{\hat{K}}{1+\hat{K} \hat{G}}\right] r(t) \\
& =-\left(\hat{S}_{u p}-\hat{\hat{S}}_{u p}\right) r(t)=\hat{S}_{y p}(K-\hat{K}) \hat{\hat{S}}_{y p} r(t)
\end{aligned}
$$

Using Parseval's relation, the asymptotic bias distribution of the parameter estimates in the frequency domain will be given by:

$$
\begin{aligned}
\hat{\theta}^{*} & =\arg \min _{\theta} \int_{-\pi}^{\pi}\left|\hat{S}_{y p}\right|^{2}|K-\hat{K}|^{2}\left|\hat{\hat{S}}_{y p}\right|^{2} \phi_{r}(\omega) d \omega \\
& =\arg \min _{\theta} \int_{-\pi}^{\pi}\left|\hat{S}_{u p}-\hat{\hat{S}}_{u p}\right|^{2} \phi_{r}(\omega) d \omega
\end{aligned}
$$

where $\hat{\theta}^{*}$ is the vector of estimated parameters of the reduced order controller and $\phi_{r}(\omega)$ is the spectral density of the excitation signal.

(2) Closed loop output matching algorithms

In this case the output of the nominal and reduced order controller can be expressed as:

$$
\begin{aligned}
& u(t)=\frac{K \hat{G}}{1+K \hat{G}} r(t) \\
& \hat{u}(t)=\frac{\hat{K} \hat{G}}{1+\hat{K} \hat{G}} r(t)
\end{aligned}
$$

The closed loop output error will be given by:

$$
\begin{aligned}
\varepsilon_{C L}(t) & =u(t)-\hat{u}(t)=\left[\frac{K \hat{G}}{1+K \hat{G}}-\frac{\hat{K} \hat{G}}{1+\hat{K} \hat{G}}\right] r(t) \\
& =\left(\hat{S}_{y p}-\hat{\hat{S}}_{y p}\right) r(t)=\hat{S}_{y p}(K-\hat{K}) \hat{\hat{S}}_{y v} r(t)
\end{aligned}
$$

Using Parseval's relation, the asymptotic bias distribution of the parameter estimates in the frequency domain will be given by:

$$
\begin{aligned}
\hat{\theta}^{*} & =\arg \min _{\theta} \int_{-\pi}^{\pi}\left|\hat{S}_{y p}\right|^{2}|K-\hat{K}|^{2}\left|\hat{\hat{S}}_{y v}\right|^{2} \phi_{r}(\omega) d \omega \\
& =\arg \min _{\theta} \int_{-\pi}^{\pi}\left|\hat{S}_{y p}-\hat{\hat{S}}_{y p}\right|^{2} \phi_{r}(\omega) d \omega
\end{aligned}
$$

where $\hat{\theta}^{*}$ is the vector of estimated parameters of the reduced order controller and $\phi_{r}$ is the spectral density of the excitation signal. 
Expressions (42) and (47) clearly show that:

- The two norm expression of either Eq. (5) for CLIM (the difference between $\hat{S}_{u p}$ and $\hat{\hat{S}}_{u p}$ ) or Eq. (8) for CLOM (the difference between $\hat{S}_{y p}$ and $\hat{\hat{S}}_{y p}$ ) are minimized when $r(t)$ is a white noise signal (since the spectral density of a white noise is a constant).

- The frequency distribution of the bias is weighted by the spectrum of the sensitivity functions of the nominal simulated system and the spectrum of the estimated sensitivity functions (given by the plant model and the estimated reduced order controller).

- The difference between $K$ and $\hat{K}$ is minimized in the critical frequency regions for control where the modulus of the sensitivity functions is large.

- The frequency distribution of the bias can be tuned by the choice of $r(t)$.

\subsubsection{Use of real data}

(1) Closed loop input matching algorithm

The same algorithm applies when real data are used (Fig. 3). In this case the expression of the closed loop error will be given by:

$$
\begin{aligned}
\varepsilon_{C L}(t) & =\left[\frac{K}{1+K G}-\frac{\hat{K}}{1+\hat{K} \hat{G}}\right] r(t)+\frac{1}{1+K G} v^{\prime}(t) \\
& =-\left(S_{u p}-\hat{\hat{S}}_{u p}\right) r(t)+S_{y p} v^{\prime}(t)
\end{aligned}
$$

where

$$
v^{\prime}(t)=v(t)-K p(t)
$$

In Eq. (50) $v(t)$ and $p(t)$ represent the input and output disturbances (noise) respectively. The asymptotic bias distribution of the parameter estimates in the frequency domain will be given by:

$$
\begin{aligned}
\hat{\theta}^{*}= & \arg \min _{\theta} \int_{-\pi}^{\pi}\left\{\left|S_{u p}-\hat{\hat{S}}_{u p}\right|^{2} \phi_{r}(\omega)+\left|S_{y p}\right|^{2} \phi_{v^{\prime}}(\omega)\right\} d \omega \\
= & \arg \min _{\theta} \int_{-\pi}^{\pi}\left\{\left|S_{y p}(K-\hat{K}) \hat{\hat{S}}_{y p}+S_{u p}(\hat{G}-G) \hat{\hat{S}}_{u p}\right|^{2} \phi_{r}(\omega)\right. \\
& \left.+\left|S_{y v}\right|^{2} \phi_{v^{\prime}}(\omega)\right\} d \omega
\end{aligned}
$$

(2) Closed loop output matching algorithms

For CLOM1, the controller output of the nominal and reduced order controller can be expressed as:

$$
u(t)=\frac{K \hat{G}}{1+K G} r(t)+\frac{1}{1+K G} v^{\prime}(t)
$$




$$
\hat{u}(t)=\frac{\hat{K} \hat{G}}{1+\hat{K} \hat{G}} r(t)
$$

The closed loop output error will be given by:

$$
\begin{aligned}
\varepsilon_{C L}(t) & =u(t)-\hat{u}(t)=\left[\frac{K \hat{G}}{1+K G}-\frac{\hat{K} \hat{G}}{1+\hat{K} \hat{G}}\right] r(t)+\frac{1}{1+K G} v^{\prime}(t)(54 \\
& =S_{y p}(K-\hat{K}) \hat{\hat{S}}_{y v} r(t)+S_{u p}(\hat{G}-G) \hat{\hat{S}}_{y r} r(t)+S_{y p} v^{\prime}(t)
\end{aligned}
$$

Using Parseval's relation again, the asymptotic bias distribution will be given by:

$$
\begin{aligned}
\hat{\theta}^{*}= & \arg \min _{\theta} \int_{-\pi}^{\pi}\left\{\left|S_{y p}(K-\hat{K}) \hat{\hat{S}}_{y v}+S_{u p}(\hat{G}-G) \hat{\hat{S}}_{y r}\right|^{2} \phi_{r}(\omega)\right. \\
& \left.+\left|S_{y p}\right|^{2} \phi_{v^{\prime}}(\omega)\right\} d \omega
\end{aligned}
$$

For CLOM2 the output of the nominal controller is given by Eq. (52) in which $\hat{G}$ is replaced by $G$. The output of the reduced order controller is given by Eq. (53). This will finally yields to:

$$
\begin{aligned}
& \hat{\theta}^{*}= \arg \min _{\hat{K}(\theta)} \int_{-\pi}^{\pi}\left\{\left|S_{y p}-\hat{\hat{S}}_{y p}\right|^{2} \phi_{r}(\omega)+\left|S_{y p}\right|^{2} \phi_{v^{\prime}}(\omega)\right\} d \omega \\
&=\arg \min _{\hat{K}(\theta)} \int_{-\pi}^{\pi}\left\{\left|\hat{\hat{S}}_{y p}(G K-\hat{G} \hat{K}) S_{y p}\right|^{2} \phi_{r}(\omega)+\left|S_{y p}\right|^{2} \phi_{v^{\prime}}(\omega)\right\} d \omega \\
&=\arg \min _{\hat{K}(\theta)} \int_{-\pi}^{\pi}\left\{\left|\hat{\hat{S}}_{u p}(G-\hat{G}) S_{y p}-\hat{\hat{S}}_{y p}(K-\hat{K}) S_{y v}\right|^{2} \phi_{r}(\omega)\right. \\
& \\
&\left.\quad+\left|S_{y p}\right|^{2} \phi_{v^{\prime}}(\omega)\right\} d \omega
\end{aligned}
$$

Expressions (51), (56) and (57) show that:

- The noise does not affect the asymptotic bias distribution of the controller parameters estimate.

- The feedback system using the reduced order controller approximates the real closed loop system instead of the nominal simulated system for CLIM and CLOM2.

- The frequency distribution of the bias can be tuned by the choice of $r(t)$.

Eq. (57) (as well as Eqs. (51) and (56)) shows that the real data allows to take into account (in the good direction) the modeling error which always exists between the plant model used for controller reduction and the true plant model. Consider that $G-\hat{G}$ is small in some frequency regions (normally at low frequencies), therefore the term $\hat{\hat{S}}_{u p}(G-\hat{G}) S_{y p}$ can be ignored and the 
minimization of the integral leads to the weighted minimization of $|K-\hat{K}|$ at low frequencies. Now consider that $|G|<<1$ in some frequency regions (normally at high frequencies), therefore the second term can be ignored and the minimization of the integral leads to the minimization of the magnitude of the input sensitivity function $\left|\hat{\hat{S}}_{u p}\right|$ at the frequencies where the modeling error $G-\hat{G}$ is large, thus improving the robustness. For an example which illustrates this property see Karimi and Landau (2000).

\section{Validation of the estimated reduced order controller}

Once a reduced order controller has been estimated, it must be validated before it is applied on the real system.

\subsection{Simulated Data}

In this case we assume that the nominal controller stabilizes both the real plant and the plant model used in the reduction procedure. In actual fact controller reduction takes place with respect to the available plant model which is assumed to correspond to the real plant model. Also any uncertainties were taken into account when the nominal controller was designed (as in all modelbased controller reduction techniques). The resulting reduced order controller should stabilize the nominal model and yield sensitivity functions which are close to nominal ones in the critical frequency regions for performance and robustness.

In many applications, the output sensitivity function and the input sensitivity function are important. Therefore, in addition to testing closed loop stability when using a reduced order controller, it is necessary to check the closeness of the nominal and reduced order sensitivity functions in the frequency domain since they are relevant both for performance and robustness.

The Vinnicombe distance ( $\nu$-gap) between the sensitivity functions obtained with the nominal and the reduced order controller, proposed in Vinnicombe (1993), uses a single number to make a first evaluation and classification of the results for various reduced order controllers. Then, a visual comparison of the frequency characteristics of the various sensitivity functions will allow us to decide whether the results obtained are satisfactory.

Another important aspect is tolerance with respect to the uncertainties between the true plant model and the nominal plant model used for design and controller reduction. If we assume that this robustness issue was taken into 
account when the nominal controller was designed, we have to try to preserve this property for the case of the reduced order controller. Comparison of the sensitivity functions already provides valuable information, but more complete results can be obtained using the Vinnicombe stability test in Vinnicombe (1993); Zhou and Doyle (1998). A controller $K_{i}$ which stabilizes the plant model $G_{1}$ will also stabilize all the plant models $G_{2}$ satisfying the condition

$$
\delta_{\nu}\left(G_{1}, G_{2}\right) \leq b\left(K_{i}, G_{1}\right)
$$

where $\delta_{\nu}\left(G_{1}, G_{2}\right)$ is the $\nu$-gap between the two plant models and $b\left(K_{i}, G_{1}\right)$ is the generalized stability margin defined as (Vinnicombe (1993); Zhou and Doyle (1998)):

$$
b\left(K_{i}, G_{1}\right)=\left\{\begin{array}{cc}
\left\|T\left(K_{i}, G_{1}\right)\right\|_{\infty}^{-1} & \text { if }\left(K_{i}, G_{1}\right) \text { is stable } \\
0 & \text { otherwise }
\end{array}\right.
$$

where

$$
T\left(K_{i}, G_{1}\right)=\left[\begin{array}{cc}
S_{y r} & S_{y v} \\
-S_{u p} & S_{y p}
\end{array}\right]
$$

and $S_{x y}$ are computed for $\left(K_{i}, G_{1}\right)$.

Therefore, in order to preserve the robustness of the reduced order controller with respect to the plant model uncertainties, we must check that $b\left(K_{i}, G_{1}\right)$ is close to $b\left(K, G_{1}\right)$, where $K$ is the nominal controller and $K_{i}$ is a reduced order controller.

\subsection{Real time data}

In this case there are two options. The first one is to validate the reduced order controller obtained with real time data using the validation methods for "simulated data" (see above). The second option is to take advantage of the available real time data. We will focus on this issue below.

With reference to Fig. 3, the objective is to test to what extent the estimated closed loop with a reduced order controller is close to the real closed loop system formed by the plant and the nominal controller. 
Basic information is provided by the variance of the residual closed loop error. Moreover, as long as the complexity of the two closed loop systems is not too different, the values of the cross-correlations between the residual closed loop error and the output of estimated controller generated in closed loop with the plant model are good indicators. This is similar to the statistical tests used in closed loop identification (see Landau and Karimi (1997b); Landau et al. (1997)).

The other alternative for validation using real data is to compute the Vinnicombe gap between the identified transfer function of the true closed loop system (between $r$ and $u$ ) which uses the nominal controller and the computed transfer function of the closed loop formed by the reduced order controller and the estimated plant model.

\section{$5 \quad$ Practical aspects}

The controller reduction problem can be approached in two ways. The first is to assume that the plant model is given and that the nominal controller has been designed allowing for both performance specification and supposed uncertainty of the model. This is the basic situation considered in all classical reduction techniques, which results in the context of this paper in performing reduction using simulated data.

However, if access to the real-system is possible and the nominal controller can be implemented, then there are two possibilities:

- improve the quality of the model by identification in closed loop (see Landau and Karimi (1997b); Landau et al. (1997));

- use real-data for controller reduction.

Once a plant model has been selected, the procedure for controller reduction is applied using real data or simulated data (with a preference for real data). The reduction procedure can scan all the orders for the polynomials $\mathrm{R}$ and $\mathrm{S}$ below the nominal ones using a single set of data. Those offering good approximation of the closed loop behaviour are selected.

\section{Identification of reduced order controllers - Experimental results}

The procedure for obtaining reduced order controllers by identification in closed loop will be illustrated for the case of control of an active suspension. The active suspension is shown in Fig. 5 and the corresponding block diagram 


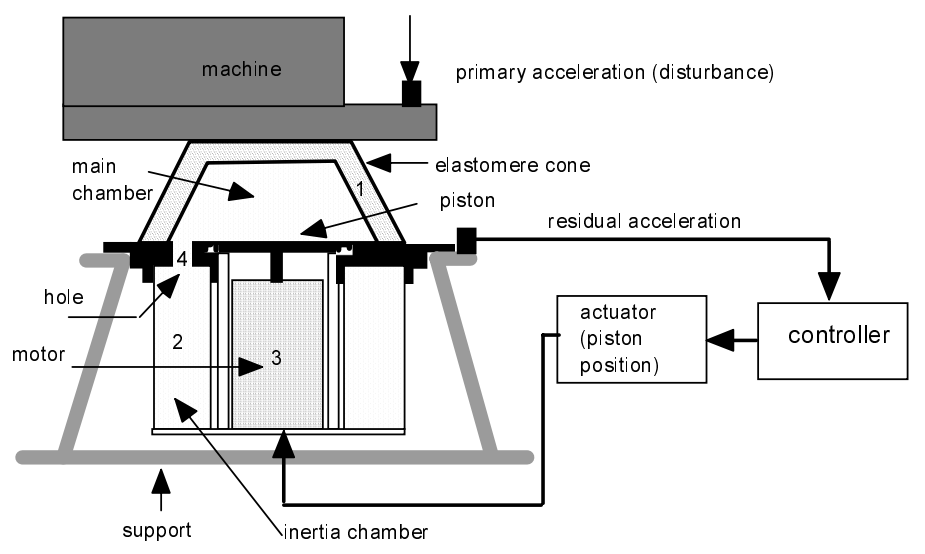

Fig. 5. The active suspension system

in Fig.6. The controller via the power amplifier will act on the piston in order to reduce the residual acceleration. The frequency spectrum of the vibrations which have to be reduced is limited by an upper frequency lower than $200 \mathrm{~Hz}$. The system is controlled by a PC via an I/O board. Sampling frequency is $800 \mathrm{~Hz}$.

The primary acceleration was generated using a shaker. Its input is a signal given by the computer. The primary path model, between the excitation of the shaker and the residual acceleration, has several vibration modes: the first is at $31.47 \mathrm{~Hz}$ with a damping factor 0.093. The goal is to compute a controller which minimizes the residual acceleration around the first vibration mode and to try to distribute the amplification of the disturbances over the high frequency range.

The identified plant model, between piston position and the residual acceleration (the secondary path), has the following complexity: $n_{B}=11, n_{A}=12$, $d=2$. The system contains a double differentiator. Fig. 7 illustrates the frequency characteristics of two models for the secondary path:

(1) The open loop identified model, used to design the nominal controller. This model was identified in open loop using a PRBS generated by a 9-bit shift register, with a clock frequency of $f s / 4$ (data length is $L=2048$ samples).

(2) The closed loop identified model, used to identify the reduced order controllers. This model gives better results in terms of closed loop validation and was identified in closed loop using the F-CLOE method (see Landau and Karimi (1997a)), the nominal controller and the same excitation signal as in open loop identification.

The results for controller reduction are given for the model identified in closed loop. The model has six vibration modes. Five of the six vibration modes have 
a very low damping $(<0.085)$. Note that an important attenuation on $S_{y p}$ is required at the frequency of the first vibration mode.

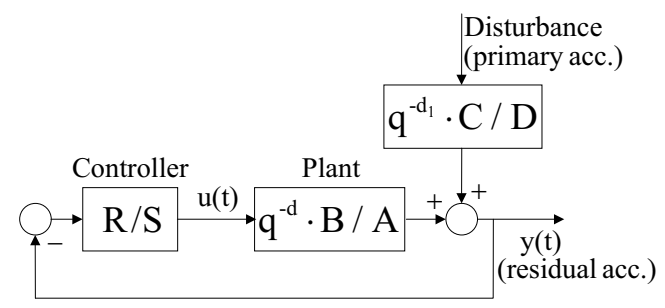

Fig. 6. Block diagram of the active suspension system

The nominal controller was designed using the "pole placement with sensitivity function shaping by convex optimization" method (see Langer and Landau (1999)). A pair of dominant poles was fixed at the frequency of the first vibration mode, with a damping $\xi=0.8$. In addition, a fixed part $H_{R}=1+q^{-1}$ was introduced in the controller $\left(R=H_{R} R^{\prime}\right)$ to ensure opening of the loop at $0.5 f_{s}$. The resulting nominal controller satisfying the residual acceleration specifications was obtained. Its complexity is given by the orders of polynomial $R$ and $S: n_{R}=27, n_{S}=28$. Note that if standard pole placement is used, by solving the Bezout equation, the result will be a controller with the orders: $n_{R}=12, n_{S}=13$ (considering the fixed part $H_{R}$ ).

First, the CLIM direct identification method for a reduced order controller was used, based on simulated data. The external input was a PRBS generated by a 10-bit shift register, with a clock frequency of $f_{s} / 2$ (data length is $L=4096$ samples). A variable forgetting factor with $\lambda_{1}=0.95$ and $\lambda_{0}=0.9$ was used $\left(\lambda_{1}(t)=\lambda_{0} \lambda_{1}(t-1)+1-\lambda_{0}\right)$.

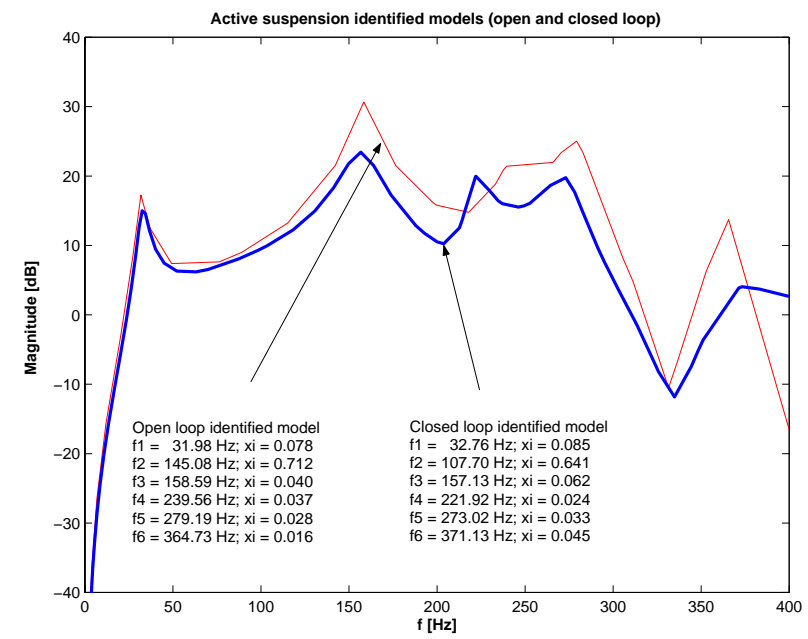

Fig. 7. The frequency characteristics of the secondary path models (input: piston displacement, output: residual acceleration) 


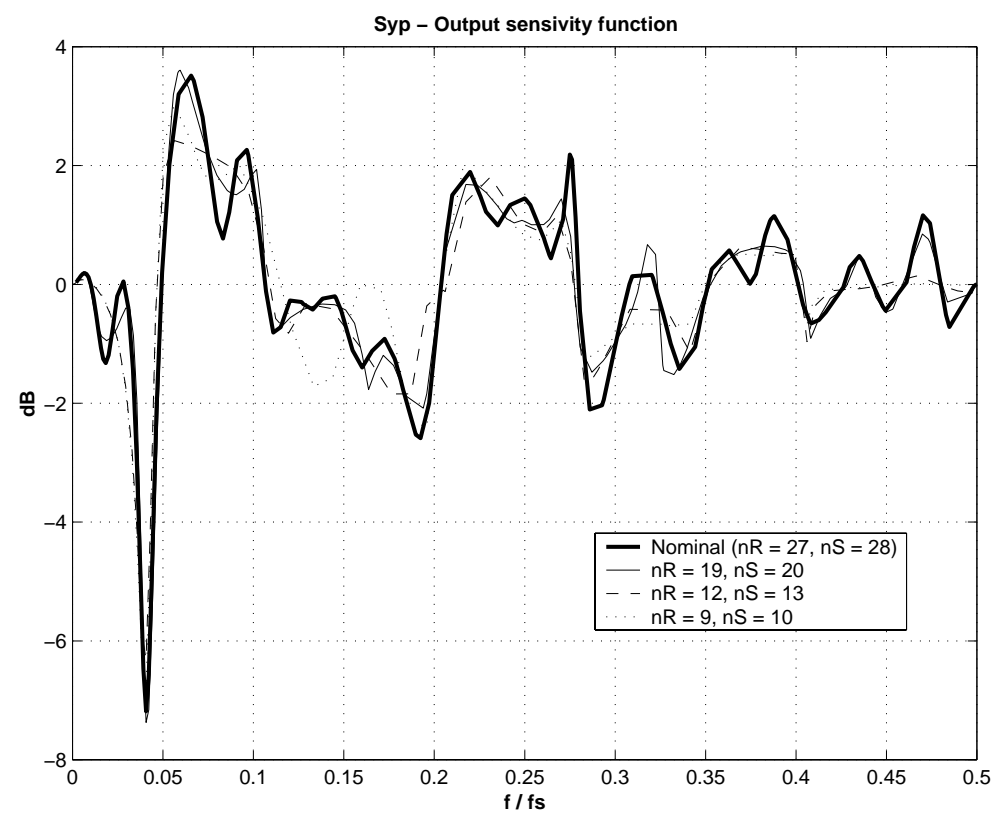

Fig. 8. Output sensitivity for the active suspension (controller reduction using CLIM algorithm on simulated data)

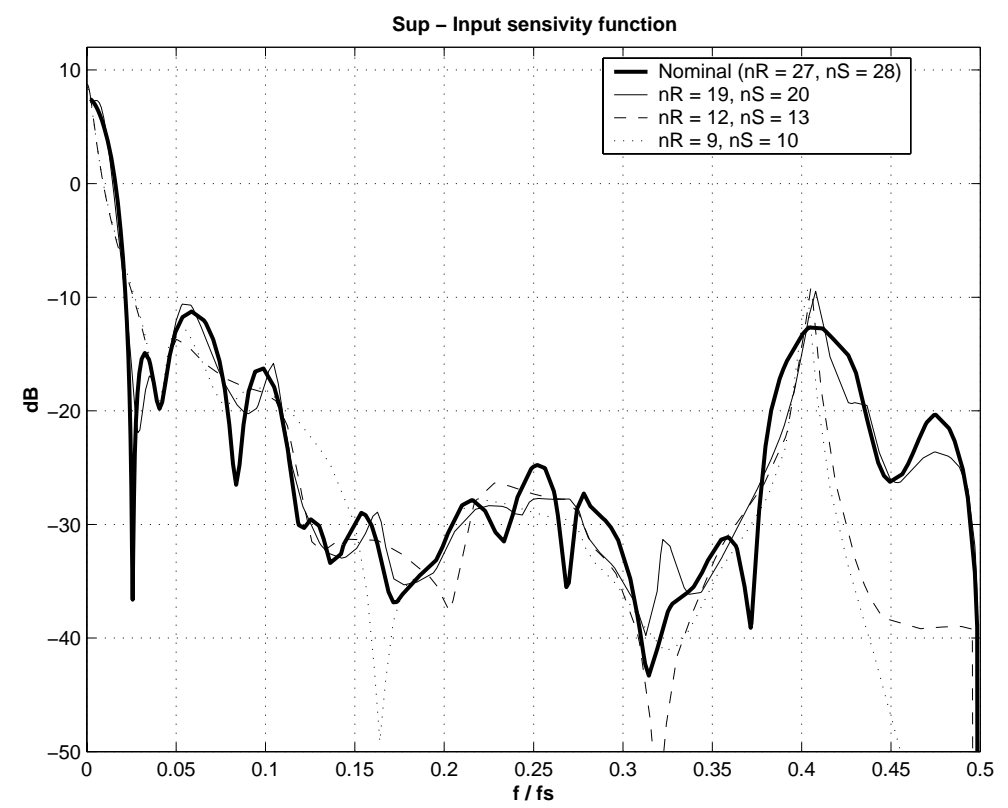

Fig. 9. Input sensitivity for the active suspension (controller reduction using CLIM algorithm on simulated data)

The frequency characteristics of the sensitivity functions $\left(S_{y p}, S_{u p}\right)$ and of the various controllers for the nominal controller $\left(K_{n}\right)$ with $n_{R}=27, n_{S}=28$, and for three reduced order controllers: $K_{1}$ with $n_{R}=19, n_{S}=20, K_{2}$ with $n_{R}=12, n_{S}=13$ and $K_{3}$ with $n_{R}=9, n_{S}=10$ are shown in figures 8 and 9 respectively (a fixed part $H_{R}=1+q^{-1}$ was imposed in the reduced order controllers). 


\begin{tabular}{|c|c|c|c|c|c|}
\hline \multicolumn{2}{|c|}{ Controller } & $\begin{array}{c}K_{n} \\
n_{R}=27 \\
n_{S}=28\end{array}$ & $\begin{array}{c}K_{1} \\
n_{R}=19 \\
n_{S}=20\end{array}$ & $\begin{array}{c}K_{2} \\
n_{R}=12 \\
n_{S}=13\end{array}$ & $\begin{array}{c}K_{3} \\
n_{R}=9 \\
n_{S}=10\end{array}$ \\
\hline \hline 1 & $\delta_{\nu}\left(K_{n}, K_{i}\right)$ & 0 & 0.1810 & 0.5049 & 0.5180 \\
\hline 2 & $\delta_{\nu}\left(S_{u p}^{n}, S_{u p}^{i}\right)$ & 0 & 0.1487 & 0.4388 & 0.4503 \\
\hline 3 & $\delta_{\nu}\left(S_{y p}^{n}, S_{y p}^{i}\right)$ & 0 & 0.0928 & 0.1206 & 0.1233 \\
\hline 4 & $b\left(K_{i}, G\right)$ & 0.0800 & 0.0786 & 0.0685 & 0.0810 \\
\hline 5 & $\delta_{\nu}\left(C L\left(K_{n}\right), C L\left(K_{i}\right)\right)$ & 0.1296 & 0.2461 & 0.5435 & 0.5522 \\
\hline 6 & C.L. error variance & 0.0023 & 0.0083 & 0.0399 & 0.0398 \\
\hline
\end{tabular}

Comparison of the various controllers (controller reduction using CLIM algorithm on simulated data)

Note that the reduced controller $K_{2}$ corresponds to the complexity of the pole placement controller with the fixed part $H_{R}$, (i.e. as a side result, the approach proposed here seeks out an optimal configuration for the closed loop poles in order to achieve the stipulated performance). Complexity of controller $K_{3}$ is lower than that corresponding to pole placement. It is also important to see the values of the various $\nu$-gap. These results are summarized in the Table 1 (the rows 1 to 3 ). The last 2 rows in Table 1 summarize real time results. We observe that the generalized stability margins $b\left(K_{i}, G\right)$ computed with the nominal model for the various reduced order controllers are close to the stability margin obtained with the nominal controller. Row 5 gives the $\nu$-gap between the input/output transfer function corresponding to the input sensitivity function $S_{u p}$ of the true closed loop system formed by the nominal designed controller with the real plant (obtained by identification) and the input/output transfer function of the simulated closed loop system $\left(\hat{\hat{S}}_{\text {up }}\right)$ formed by the various controllers (including the nominal one and the reduced ones obtained using simulated data) in feedback connection with the plant model. This quantity is denoted by $\delta_{\nu}\left(C L\left(K_{n}\right), C L\left(K_{i}\right)\right)$. This is a good criterion for validation of the reduced order controller. Row 6 gives the variance of the residual closed loop input error between the true system and the simulated one. We observe that the $\nu-$ gap and the closed loop error variance have a coherent evolution.

To illustrate the performance of the resulting controllers $\left(K_{n}, K_{1}, K_{2}\right.$ and $K_{3}$ ) on the real system, the spectral density of the residual acceleration in open and in closed loop is shown in Fig. 10. The primary source of vibration (shaker's excitation) is a PRBS. The characteristics obtained in closed loop operation with the four controllers are compared with the characteristic of the open loop operation (the interesting frequency range is 0 to $0.25 f_{s}(200 \mathrm{~Hz})$ ). 


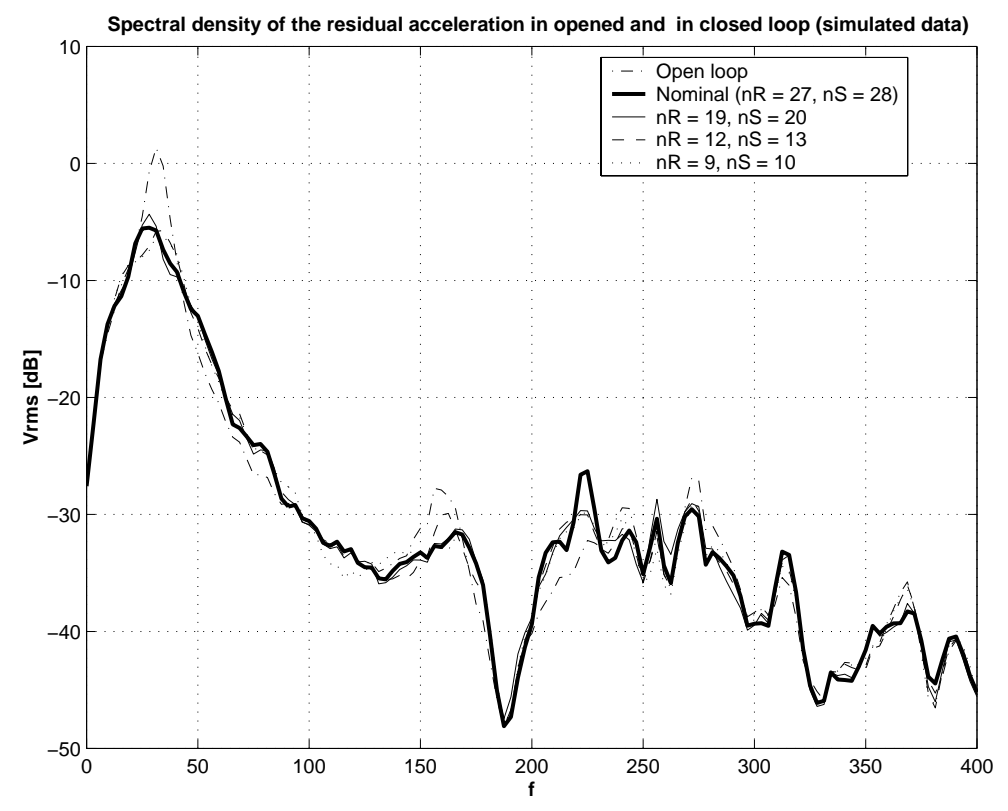

Fig. 10. Spectral density of the residual acceleration in open and in closed loop (controller reduction using CLIM algorithm on simulated data)

We note that the performance of the reduced order controllers is similar to that of the nominal controller and that they all achieve a significant reduction of the vibrations around the first vibration mode of the plant model.

The results obtained using the real data (Landau et al. (2001)) show that the performance of the reduced order controllers is very similar to the case with the simulated data. This can be accounted for by the quality of the identified model used for controller reduction.

Good results have been obtained also with the CLOM algorithms (Landau et al. (2001)) using simulated and real data. Comparison of the results obtained with CLIM and CLOM algorithms shows that the $\nu$-gap between the nominal and the reduced order output sensitivity function $\delta_{\nu}\left(S_{y p}^{n}, S_{y p}^{i}\right)$ for CLOM algorithms is slightly smaller, while the $\nu$-gap between the nominal and reduced order input sensitivity $\delta_{\nu}\left(S_{u p}^{n}, S_{u p}^{i}\right)$ for CLOM algorithms is larger. This is in agreement with the bias analysis, since the CLIM algorithm gives preference to minimizing the error between the input sensitivity functions while the CLOM algorithm gives preference to minimizing the error between the output sensitivity functions.

\section{Conclusions}

Algorithms for direct closed loop identification of reduced order digital controllers have been proposed, analyzed and experimentally tested. These al- 
gorithms are the dual of closed loop output error identification algorithms. The algorithms try to minimize either the error between inputs generated in closed loop operation by the nominal controller and the estimated reduced order controller, or the closed loop output error between the two loops.

The possibility of using real data for estimation of reduced order controllers makes it possible to take into account to a certain extent in the reduction procedure, the discrepancies between the true plant and the plant model used for design and controller reduction.

While the specific algorithms proposed have a recursive form, it is also possible to develop iterative algorithms along the lines used for closed loop identification (see Van Donkelaar and Van den Hof (2000)). Unfortunately this will raise similar theoretical problems.

Validation tests related to the $\nu$-gap and Vinnicombe stability test have been proposed. Use of the results given in Vinnicombe $(1993,1996)$; Anderson and Gevers (1998) for reduced order controller validation deserve further investigation. Future work should consider the extension to the multivariable case.

\section{Acknowledgements}

The authors would like to thank one of the anonymous reviewers for his suggestions concerning the interpretation of the validation tests.

\section{References}

[1] Anderson, B., Gevers, M., 1998. Fundamental problems in adaptive control. In: Normand-Cyrot, D. (Ed.), Perspectives in Control. Springer-Verlag, London, UK.

[2] Anderson, B. D. O., 1986. Weighted Hankel norm approximation: Calculation of bounds. Systems and Control Letters 7 (4), 247-255.

[3] Anderson, B. D. O., August 1993. Controller design: Moving from theory to practice. IEEE Control Magazine .

[4] Anderson, B. D. O., Liu, Y., August 1989. Controller reduction: Concepts and approaches. IEEE Transactions on Automatic Control 34 (8), 802-812.

[5] Bendotti, P., Cordons, B., Falinower, C., Gevers, M., December 1998. Control oriented low order modeling of a complex PWR plant: a comparison between open loop and closed loop methods. In: IEEE-CDC 1998 (FAO 7-2). Tampa, Florida USA.

[6] Cordons, B., Bendotti, P., Falinower, C., Gevers, M., December 1999. A com- 
parison between model reduction and controller reduction: Application to a PWR nuclear plant. In: IEEE-CDC. ix, USA, pp. 4625-4630.

[7] Enns, D., December 1984. Model reduction with balanced realization: An error bound and a frequency weighted generalization. In: 23-rd CDC. Las Vegas, Nevada USA, pp. 127-132.

[8] Hjalmarsson, H., Gevers, M., Gunnarsson, S., Lequin, O., 1998. Iterative feedback tuning: Theory and application. IEEE Control Systems Magazine , $26-41$.

[9] Hjalmarsson, H., Gunnarsson, S., Gevers, M., December 1994. A convergent iterative restricted complexity control design scheme. In: 33rd IEEE-CDC.

[10] Karimi, A., Landau, I. D., June 2000. Controller order reduction by direct closed loop identification (output matching). In: 3rd Rocond IFAC. Prague.

[11] Landau, I. D., Karimi, A., May 1997a. An output error recursive algorithm for unbiased identification in closed loop. Automatica 33 (5), 933-938.

[12] Landau, I. D., Karimi, A., August 1997b. Recursive algorithms for identification in closed loop - a unified approach and evaluation. Automatica 33 (8), 1499-1523.

[13] Landau, I. D., Karimi, A., December 2000. Model estimation and controller reduction: Dual closed loop identification problems. In: 39-th IEEE-CDC. Sydney.

[14] Landau, I. D., Karimi, A., Constantinescu, A., 2001. Direct controller order reduction by identification in closed-loop - application to active suspension control. Tech. rep., Laboratoire d'Automatique de Grenoble, ENSIEG, BP. 46, 38402 St. Martin d'Heres.

[15] Landau, I. D., Lozano, R., M'Saad, M., 1997. Adaptive Control. Springer Verlag, London.

[16] Landau, I. D., Rey, D., Karimi, A., Voda, A., Franco, A., 1995. A flexible transmission system as a benchmark for robust digital control. European Journal of Control 1 (2).

[17] Langer, J., Landau, I., 1999. Combined pole placement/sensitivity function shaping method using convex optimization criteria. Automatica 35, 11111120.

[18] Van Donkelaar, E. T., Van den Hof, P. M. J., 2000. Analysis of closed-loop identification with tailor-made parameterization. European Journal of Control 6 (1), 54-62.

[19] Vinnicombe, G., 1993. Frequency domain uncertainty and the graph topology. IEEE Trans. on Automatic Control 38, 1571-1583.

[20] Vinnicombe, G., 1996. The robustness of feedback systems with bounded complexity. IEEE Trans. on Automatic Control 41 (6), 1571-1583.

[21] Zhou, K., Doyle, J. C., 1998. Essentials of robust control. Prentice-Hall, N.Y. 